\title{
A suffix tree approach to anti-spam email filtering
}

\author{
Rajesh Pampapathi · Boris Mirkin • Mark Levene
}

Received: 3 March 2005 / Revised: 1 June 2006 / Accepted: 12 June 2006 / Published online: 27 July 2006

Springer Science + Business Media, LLC 2006

\begin{abstract}
We present an approach to email filtering based on the suffix tree data structure. A method for the scoring of emails using the suffix tree is developed and a number of scoring and score normalisation functions are tested. Our results show that the character level representation of emails and classes facilitated by the suffix tree can significantly improve classification accuracy when compared with the currently popular methods, such as naive Bayes. We believe the method can be extended to the classification of documents in other domains.
\end{abstract}

Keywords Suffix tree $\cdot$ Spam $\cdot$ E-mail filtering $\cdot$ Scoring function $\cdot$ Text categorization

\section{Introduction}

Just as email traffic has increased over the years since its inception, so has the proportion that is unsolicited; some estimations have placed the proportion as high as $60 \%$, and the average cost of this to business at around $\$ 2000$ per year, per employee (see (unspam.com, 2004) for a range of numbers and statistics on spam). Unsolicited emails-commonly known as spam - have thereby become a daily feature of every email user's inbox; and regardless of advances in email filtering, spam continues to be a problem in a similar way to computer viruses which constantly reemerge in new guises. This leaves the research community with the task of continually investigating new approaches to sorting the welcome emails (known as ham) from the unwelcome spam.

Editor: Tom Fawcett

R. Pampapathi $(\bowtie) \cdot$ B. Mirkin · M. Levene

School of Computer Science and Information Systems, Birkbeck College, University of London e-mail: rajesh@dcs.bbk.ac.uk

B. Mirkin

e-mail: mirkin@dcs.bbk.ac.uk

M. Levene

e-mail: mark@dcs.bbk.ac.uk 
We present just such an approach to email classification and filtering based on a well studied data structure, the suffix tree (see (Lloyd, 2000) for a brief introduction). The approach is similar to many existing ones, in that it uses training examples to construct a model or profile of the class and its features, then uses this to make decisions as to the class of new examples; but it differs in the depth and extent of the anaysis. For a good overview of a number of text classification methods, see (Sebastiani, 2002; Aas \& Eikvil, 1999; Weiss et al., 2005).

Using a suffix tree, we are able to compare not only single words, as in most current approaches, but substrings of an arbitrary length. Comparisons of substrings (at the level of characters) has particular benefits in the domain of spam classification because of the methods spammers use to evade filters. For example, they may disguise the nature of their messages by interpolating them with meaningless characters, thereby fooling filters based on keyword features into considering the words, sprinkled with random characters, as completely new and unencountered. If we instead treat the words as character strings, and not features in themselves, we are still able to recognise the substrings, even if the words are broken.

Section 2 gives examples of some of the methods spammers use to evade detection which make it useful to consider character level features. Section 3 gives a brief explanation of the naive Bayes method of text classification as an example of a conventional approach. Section 4 briefly introduces suffix trees, with some definitions and notations which are useful in the rest of the paper, before going on to explain how the suffix tree is used to classify text and filter spam. Section 5 describes our experiments, the test parameters and details of the data sets we used. Section 6 presents the results of the experiments and provides a comparison with results in the literature. Section 7 concludes.

\section{Examples of spam}

Spam messages typically advertise a variety of products or services ranging from prescription drugs or cosmetic surgery to sun glasses or holidays. But regardless of what is being advertised, one can distinguish between the methods used by the spammer to evade detection. These methods have evolved with the filters which attempt to intercept them, so there is a generational aspect to them, with later generations becoming gradually more common and earlier ones fading out; as this happens, earlier generations of filters become less effective.

We present four examples of spam messages, the first of which illustrates undisguised spam while the other three illustrate one or more methods of evasion.

1. Undisguisedmessage. The example contains no obfuscation. The content of the message is easily identified by filters, and words like "Viagra" allow it to be recognised as spam. Such messages are very likely to be caught by the simplest word-based Bayesian classifiers.

Buy cheap medications online, no prescription needed.

We have Viagra, Pherentermine, Levitra, Soma, Ambien, Tramadol and many more products.

No embarrasing trips to the doctor, get it delivered directly to your door.

Experienced reliable service.

Most trusted name brands.

Your solution is here: http://www.webrx-doctor.com/?rid=1000 


\section{Intra-word characters.}

Get the low.est pri.ce for gen.eric medica.tions!

Xa.n.ax - only $\$ 100$

Vi.cod.in - only $\$ 99$

Ci.al.is - only $\$ 2$ per do.se

Le.vit.ra - only $\$ 73$

Li.pit.or - only $\$ 99$

Pr.opec.ia - only $\$ 79$

Vi.agr.a - only $\$ 4$ per do.se

Zo.co.r - only $\$ 99$

Your Sav.ings $40 \%$ compared Average Internet Pr.ice!

No Consult.ation Fe.es! No Pr.ior Prescrip.tions Required! No Appoi.ntments!

No Wait.ing Room! No Embarra.ssment! Private and Confid.ential! Disc.reet

Packa.ging!

che ck no w:

<http://priorlearndiplomas.com/r3/?d=getanon>

The example above shows the use of intra-word characters, which may be non-alphanumeric or whitespace. Here the word "Viagra" has become "Vi.agr.a", while the word "medications" has become "medica.tions". To a simple word-based Bayesian classifier, these are completely new words, which might have occurred rarely, or not at all, in previous examples. Obviously, there are a large number of variations on this theme which would each time create an effectively new word which would not be recognised as spam content. However, if we approach this email at the character level, we can still recognise strings such as "medica" as indicative of spam, regardless of the character that follows, and furthermore, though we do not deal with this in the current paper, we might implement a look-ahead window which attempts to skip (for example) non-alphabetic characters when searching for spammy features.

Certainly, one way of countering such techniques of evasion is to map the obfuscated words to genuine words during a pre-processing stage, and doing this will help not only word-level filters, but also character-level filters because an entire word match, either as a single unit, or a string of characters, is better than a partial word match $^{1}$.

\footnotetext{
${ }^{1}$ The subject of spam de-obfuscation has been addressed in a recent paper: Honglak Lee and Andrew Y. Ng, Spam deobfuscation using a hidden Markov model, Proceedings of the Second Conference on Email and Anti-Spam (CEAS, 2005), Stanford University, CA.
} 
However, some other methods may not be evaded so easily in the same way, with each requiring its own special treatment; we give two more examples below which illustrate the point.

3. Word salad.

Buy meds online and get it shipped to your door Find out more here $<$ http: / / www. gowebrx.com/ ?rid=1001>

a publications website accepted definition. known are can Commons the be definition. Commons UK great public principal work Pre-Budget but an can Majesty's many contains statements statements titles (eg includes have website. health, these Committee Select undertaken described may publications

The example shows the use of what is sometimes called a word salad-meaning a random selection of words. The first two lines of the message are its real content; the paragraph below is a paragraph of words taken randomly from what might have been a government budget report. The idea is that these words are likely to occur in ham, and would lead a traditional algorithm to classify this email as such. Again, approaching this at the character level can help. For example, say we consider strings of length 8 , strings such as "are can" and "an can", are unlikely to occur in ham, but the words "an", "are" and "can" may occur quite frequently. Of course, in most 'bag-of-words' implementations, words such as these are pruned from the feature set, but the argument still holds for other bigrams.

4. Embedded message (also contains a word/letter salad). The example below shows an embedded message. Inspection of it will reveal that it is actually offering prescription drugs. However, there are no easily recognised words, except those that form the word salad, this time taken from what appear to be dictionary entries under ' $z$ '. The value of substring searching is highly apparent in this case as it allows us to recognise words such as "approved", "Viagra" and "Tablets", which would otherwise be lost among the characters pressed up against them.

These examples are only a sample of all the types of spam that exist. For an excellent and often updated list of examples and categories, see (Graham-Cummings, 2004; de Freitas \& Levene, 2004). Under the categories suggested in Wittel \& Wu (2004), example 2 and 4 would count as 'Tokenisation' and/or 'Obfuscation', while examples 2 and 3 would count as 'Statistical'.

We look next at a bag-of-words approach, naive Bayes, before considering the suffix tree approach. 
zygotes zoogenous zoometric zygosphene zygotactic zygoid zucchettos zymolysis zoopathy zygophyllaceous zoophytologist zygomaticoauricular zoogeologist zymoid zoophytish zoospores zygomaticotemporal zoogonous zygotenes zoogony zymosis zuza zoomorphs zythum zoonitic zyzzyva zoophobes zygotactic zoogenous zombies zoogrpahy zoneless zoonic zoom zoosporic zoolatrous zoophilous zymotically zymosterol

\section{FreeHYSHKRODMonthQGYIHOCSupply.IHJBUMDSTIPLIBJT}

\section{* GetJIIXOLDViagraPWXJXFDUUTabletsNXZXVRCBX <http: / /healthygrow.biz/index.php?id=2>}

zonally zooidal zoospermia zoning zoonosology zooplankton zoochemical zoogloeal zoological zoologist zooid zoosphere zoochemical

\section{\& Safezoonal andNGASXHBPnatural \\ \& TestedQLOLNYQandEAVMGFCapproved}

zonelike zoophytes zoroastrians zonular zoogloeic zoris zygophore zoograft zoophiles zonulas zygotic zymograms zygotene zootomical zymes zoodendrium zygomata zoometries zoographist zygophoric zoosporangium zygotes zumatic zygomaticus zorillas zoocurrent zooxanthella zyzzyvas zoophobia zygodactylism zygotenes zoopathological noZFYFEPBmas <http: / / heal thygrow.biz/remove.php>

\section{Naive Bayesian classification}

Naive Bayesian (NB) email filters currently attract a lot of research and commercial interest, and have proved highly successful at the task; Sahami et al. (1998) and Michelakis et al. (2004) both provide excellent studies of this approach to email filtering. We do not give detailed attention to NB as it is not the intended focus of this paper; for a general discussion of NB see Lewis (1998), for more context in text categorisation see Sebastiani (2002), and for an extension of NB to the classification of structured data, see (Flach \& Lachiche, 2004). However, an NB classifier is useful in our investigation of the suffix tree classifier, and in particular, our own implementation of NB is necessary to investigate experimental conditions which have not been explored in the literature. We therefore briefly present it here.

We begin with a set of training examples with each example document assigned to one of a fixed set of possible classes, $\mathbf{C}=\left\{\mathrm{c}_{1}, \mathrm{c}_{2}, \mathrm{c}_{3}, \ldots \mathrm{c}_{J}\right\}$. An NB classifier uses this training data to generate a probabilistic model of each class; and then, given a new document to classify, it uses the class models and Bayes' rule to estimate the likelihood with which each class generated the new document. The document is then assigned to the most likely class. The features, or parameters, of the model are usually individual words; and it is 'naive' because of the simplifying assumption that, given a class, each parameter is independent of the others.

McCallum and Nigam (1998) distinguish between two types of probabilistic models which are commonly used in NB classifiers: the multi-variate Bernoulli event model and 
the multinomial event model. We adopt the latter, under which a document is seen as a series of word events and the probability of the event given a class is estimated from the frequency of that word in the training data of the class.

Hence, given a document $\mathbf{d}=\left\{d_{1} d_{2} d_{3} \ldots d_{L}\right\}$, we use Bayes' theorem to estimate the probability of a class, $c_{j}$ :

$$
P\left(c_{j} \mid \mathbf{d}\right)=\frac{P\left(c_{j}\right) P\left(\mathbf{d} \mid c_{j}\right)}{P(\mathbf{d})}
$$

Assuming that words are independent given the category, this leads to:

$$
P\left(c_{j} \mid \mathbf{d}\right)=\frac{P\left(c_{j}\right) \prod_{i=1}^{L} P\left(d_{i} \mid c_{j}\right)}{P(\mathbf{d})}
$$

We estimate $\mathrm{P}\left(\mathrm{c}_{j}\right)$ as:

$$
\hat{P}\left(C=c_{j}\right)=\frac{N_{j}}{N}
$$

and $\mathrm{P}\left(\mathrm{d}_{i} \mid \mathrm{c}_{j}\right)$ as:

$$
\hat{P}\left(d_{i} \mid c_{j}\right)=\frac{1+N_{i j}}{M+\sum_{k=1}^{M} N_{k j}}
$$

where $N$ is the total number of training documents, $N_{j}$ is the total number of training documents belonging to class $j, N_{i j}$ is the number of times word $i$ occurs in class $j$ (similarly for $N_{k j}$ ) and $M$ is the total number of words considered.

To classify a document we calculate two scores, for spam and ham, and take the ratio, $h s r=\frac{\text { hamScore }}{\text { spamScore }}$, and classify the document as ham if it is above a threshold, $t h$, and as spam if it is below (see Section 5.1.3).

\section{Suffix tree classification}

\subsection{Introduction}

The suffix tree is a data storage and fast search technique which has been used in fields such as computational biology for applications such as string matching applied to DNA sequences (Bejerano and Yona, 2001; Lu \& Chen, 2003). To our knowledge it has not been used in the domain of natural language text classification.

We adopted a conventional procedure for using a suffix tree in text classification. As with $\mathrm{NB}$, we take a set of documents $\mathbf{D}$, each of which is known to belong to one class, $c_{j}$, in a set of classes, $\mathbf{C}$, and build one tree for each class. Each of these trees is then said to represent (or profile) a class (a tree built from a class will be referred to as a "class tree").

Given a new document $\mathbf{d}$, we score it with respect to each of the class trees and the class of the highest scoring tree is taken as the class of the document.

We address the scoring of documents in Section 4.4, but first, we consider the construction of the class tree. 


\subsection{Suffix tree construction}

We provide a brief introduction to suffix tree construction. For a more detailed treatment, along with algorithms to improve computational efficiency, the reader is directed to Gusfield (1997). Our representation of a suffix tree differs from the literature in two ways that are specific to our task: first, we label nodes and not edges, and second, we do not use a special terminal character. The former has little impact on the theory and allows us to associate frequencies directly with characters and substrings. The latter is simply because our interest is actually focused on substrings rather than suffixes; the inclusion of a terminal character would therefore not aid our algorithms, and its absence does not hinder them. Furthermore, our trees are depth limited, and so the inclusion of a terminal character would be meaningless in most situations.

Suppose we want to construct a suffix tree from the string, $s=$ "meet". The string has four suffixes: $s(1)=$ "meet", $s(2)=$ "eet", $s(3)=$ "et", and $s(4)=$ " $t$ ".

We begin at the root of the tree and create a child node for the first character of the suffix $s(1)$. We then move down the tree to the newly created node and create a new child for the next character in the suffix, repeating this process for each of the characters in this suffix. We then take the next suffix, $s(2)$, and, starting at the root, repeat the process as with the previous suffix. At any node, we only create a new child node if none of the existing children represents the character we are concerned with at that point. When we have entered each of the suffixes, the resulting tree looks like that in Fig. 1. Each node is labelled with the character it represents and its frequency. The node's position also represents the position of the character in the suffix, such that we can have several nodes labelled with the same character, but each child of each node (including the root) will carry a character label which is unique among its siblings.

If we then enter the string, $t=$ "feet", into the tree in Fig. 1, we obtain the tree in Fig. 2. The new tree is almost identical in structure to the previous one because the suffixes of the two strings are all the same but for $t(1)=$ "feet", and as we said before, we need only create a new node when an appropriate node does not already exist, otherwise, we need only increment the frequency count.

Thus, as we continue to add more strings to the tree, the number of nodes in the tree increases only if the new string contains substrings which have not previously been encountered. It follows that given a fixed alphabet and a limit to the length of substrings we consider, there is a limit to the size of the tree. Practically, we would expect that, for most classes, as we continue to add strings to the class tree, the tree will increase in size at a decreasing rate, and will quite likely stabilise.

\subsection{Class trees and their characteristics}

For any string $s$ we designate the $i^{\text {th }}$ character of $s$ by $s_{i}$; the suffix of $s$ beginning at the $i^{\text {th }}$ character by $s(i)$; and the substring from the $i^{\text {th }}$ to the $j^{\text {th }}$ character inclusively by $s(i, j)$.

Any node, $n$, labeled with a character, $c$, is uniquely identified by the path from the root to $n$. For example, consider the tree in Fig. 2. There are several nodes labelled with a " $t$ ", but we can distinguish between node $n=(" t$ " given "mee" $)=(t \mid$ mee $)$ and $p=$ (" $t$ " given "ee") $=(t \mid e e)$; these nodes are labelled $n$ and $p$ in Fig. 2 . We say that the path of $n$ is $\vec{P}_{n}=$ " $m e e$ ", and the path of $p$ is $\vec{P}_{p}=$ "ee"; furthermore, the frequency of $n$ is 1 , whereas the frequency of $p$ is 2 ; and saying $n$ has a frequency of 1 is equivalent to saying the frequency of " $t$ " given "mee" is 1 , and similarly for $p$. 
Fig. 1 A Suffix Tree after the insertion of "meet"
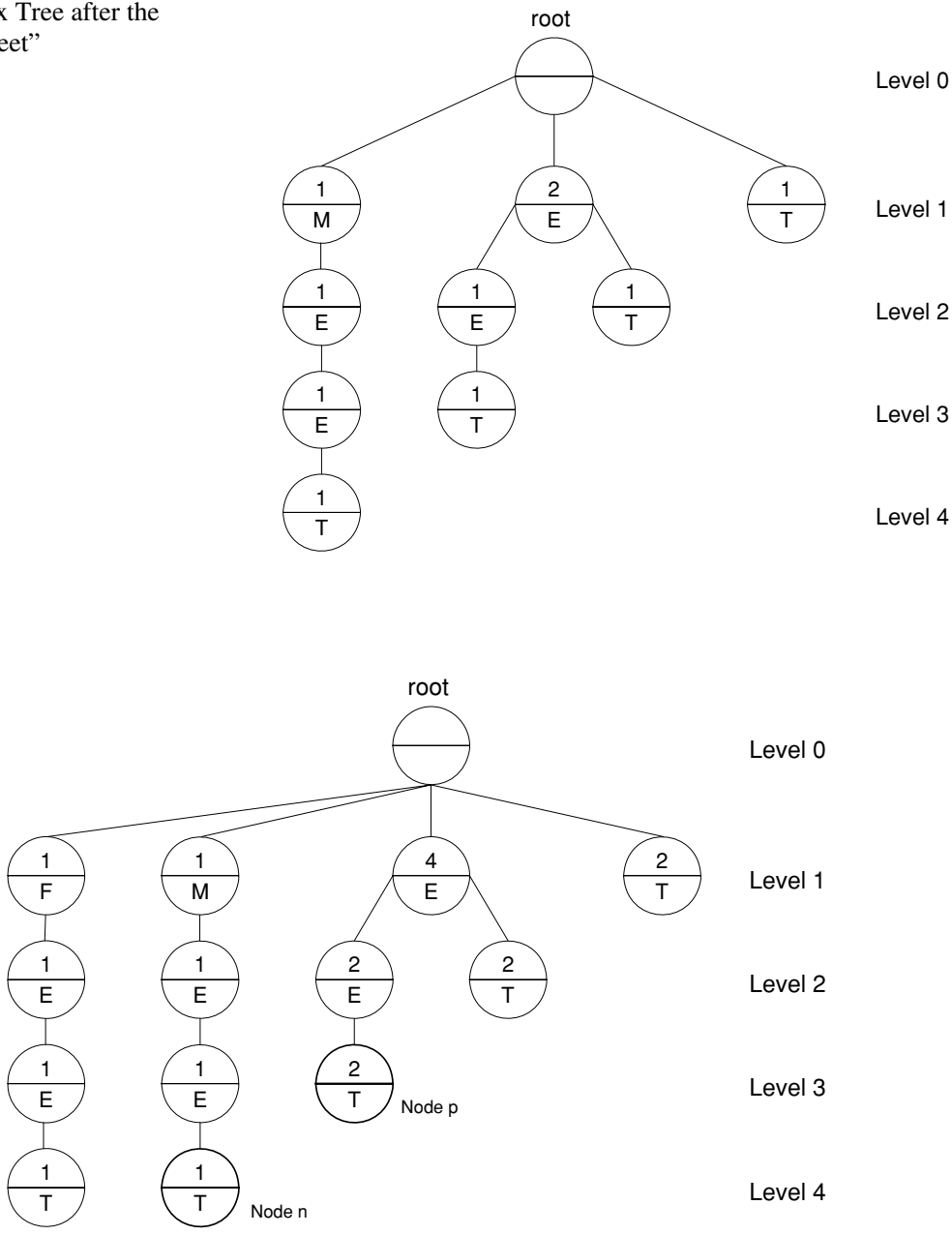

Fig. 2 A Suffix Tree after insertion of strings "meet" and "feet"

If we say that the root node, $r$, is at level zero in the tree, then all the children of $r$ are at level one. More generally, we can say that the level of any node in the tree is one plus the number of letters in its path. For example, level $(n)=4$ and level $(p)=3$.

The set of letters forming the first level of a tree is the alphabet, $\Sigma$ - meaning that all the nodes of the tree are labelled with one of these letters. For example, considering again the tree in Fig. 2, its first level letters are the set, $\Sigma=\{m, e, t, f\}$, and all the nodes of the tree are labelled by one of these.

Suppose we consider a class, $C$, containing two strings (which we might consider as documents), $s=$ "meet" and $t=$ "feet". Then we can refer to the tree in Fig. 2 as the class tree of $C$, or the suffix tree profile of $C$; which we denote by $T_{C}$.

The size of the tree, $\left|T_{C}\right|$, is the number of nodes it has not counting the root node, and it has as many nodes as $C$ has unique substrings. For instance, in the case of the tree in 
Fig. 2:

$$
\begin{aligned}
U C & =\text { uniqueSubstrings }(C)=\left\{\begin{array}{l}
\text { meet, mee, me, m, eet, ee, e, et, t, } \\
\text { feet, fee, fe, f }
\end{array}\right\} \\
|U C| & =\mid \text { uniqueSubstrings }(C) \mid=13 \\
\left|T_{C}\right| & =\text { numberOfNodes }\left(T_{C}\right)=13
\end{aligned}
$$

This is clearly not the same as the total number of substrings in $C$ :

$$
A C=\text { allsubstrings }(C)=\left\{\begin{array}{l}
\text { meet, mee, me, m, eet, ee, e, et, e, t } \\
\text { feet, fee, fe, f, eet, ee, e, et, e, t }
\end{array}\right\}
$$

$$
|A C|=\mid \text { Allsubstrings }(C) \mid=20
$$

As an example, note that the four "e"s in the set are in fact the substrings $s(1,1), s(2,2)$, $\mathrm{t}(1,1)$ and $\mathrm{t}(2,2)$.

Furthermore, as each node in the tree, $T_{C}$, represents one of the substrings in UC, the size of the class, $\mathrm{AC}$, is equal to the sum of the frequencies of nodes in the tree $T_{C}$.

$$
|A C|=\mid \text { allsubstrings }(C) \mid=\operatorname{sumofFrequencies}\left(T_{C}\right)=20
$$

In a similar way, the suffix tree allows us to read off other frequencies very quickly and easily. For example, if we want to know the number of characters in the class $C$, we can sum the frequencies of the nodes on the first level of the tree; and if we want to know the number of substrings of length 2, we can sum the frequencies of the level two nodes; and so on.

This also allows us to very easily estimate probabilities of substrings of any length (up to the depth of the tree), or of any nodes in the tree. For example, we can say from the tree in Fig. 2, that the probability of a substring, $u$, of length two, having the value, $u=$ " $e e$ ", given the class $C$, is the frequency, $f$, of the node $n=(e \mid e)$, divided by the sum of the frequencies of all the level two nodes in the tree $T_{C}$ :

$$
\text { estimatedTotalProbability }(u)=\frac{f(u)}{\sum_{i \in N_{u}} f(i)}
$$

where $N_{u}$ is the set of all nodes at same level as $u$.

Similarly one can estimate the conditional probability of $u$ as the frequency of $u$ divided by the sum of the frequencies of all the children of $u$ 's parent:

$$
\text { estimatedConditionalProbability }(u)=\frac{f(u)}{\sum_{i \in n_{u}} f(i)}
$$

where $n_{u}$ is the set of all children of $u$ 's parent.

Throughout this paper, whenever we mention $\hat{p}(u)$, we mean the second of these (formula 6): the conditional probability of a node $u$. 


\subsection{Classification using Suffix Trees}

Researchers have tackled the problem of the construction of a text classifier in a variety of different ways, but it is popular to approach the problem as one that consists of two parts:

1. The definition of a function, $C S_{i}: D \rightarrow \mathbb{R}$, where $D$ is the set of all documents; such that, given a particular document, $d$, the function returns a category score for the class $i$. The score is often normalised to ensure that it falls in the region $[0,1]$, but this is not strictly necessary, particularly if one intends, as we do, simply to take as the class prediction the highest scoring class (see Part 2 below). The interpretation of the meaning of the function, $C S$, depends on the approach adopted. For example, as we have seen, in naive Bayes, $C S(d)$, is interpreted as a probability; whereas in other approaches such as Rocchio (1971), $C S(d)$ is interpreted as a distance or similarity measure between two vectors.

2. A decision mechanism which determines a class prediction from a set of class scores. For example, the highest scoring class might be taken as the predicted class: $P C=$ $\operatorname{argmax}_{c_{j} \in C}\left\{C S_{j}(d)\right\}$. Alternatively, if $C S(d)$ is interpreted as a value with definite range, such as a probability, the decision may be based on a threshold, th, such that the predicted class is taken as $c_{j}$ if $C S_{j}(d)>$ th, and as not $c_{j}$ otherwise.

Lewis et al. (1996) and Duda and Hart (1973) refer to probabilistic models such as naive Bayes as parametric classifiers because they attempt to use the training data to estimate the parameters of a probability distribution, and assume that the estimated distribution is correct. Non-parametric, geometric models, such as Rocchio (1971), instead attempt to produce a profile or summary of the training data and use this profile to query new documents to decide their class.

It is possible to approach the construction of a suffix tree classifier in either of these two ways and indeed a probability-based approach has been developed by Bejerano and Yona (2001) for use in gene sequence matching. However, Bejerano \& Yona (2001) did not find the suffix tree entirely convenient for developing a probabilistic framework and instead developed a probabilistic analogue to the suffix tree and used this modified data structure to develop probabilistic matching algorithms.

In this paper, we retain the original structure of the suffix tree and favour a non-parametric, or geometric, approach to classifier construction. In such a framework a match between a document and a suffix tree profile of a class is a set of coinciding substrings each of which must be scored individually so that the total score is the sum of individual scores. This is analogous to the inner product between a document vector and class profile vector in the Rocchio algorithm (Rocchio, 1971). We did experiment with probabilistic models, and found that it was possible to construct one without altering the structure of the suffix tree (indeed, some of the flavours of the scoring system we present can be seen as approximating a probabilistic approach (see Section 4.4.1, Part 1C)) even though the branches are not independent: each corresponds to a set of strings which may overlap. However we found that additive scoring algorithms performed better and in the current paper we describe only this approach. The method, the details of which are presented in the next section, is governed by two heuristics:

H1 Each substring $s(i)$ that a string $s$ has in common with a class $T$ indicates a degree of similarity between $s$ and $T$, and the longer the common substrings the greater the similarity they indicate. 
H2 The more diverse ${ }^{2}$ a class $T$, the less significant is the existence of a particular common substring $s(i, j)$ between a string $s$ and the class $T$.

Turning to the second issue in classifier construction, for our current two-class problem, we take the ratio of two scores, $h s r=\frac{\text { ham Score }}{\text { spamScore }}$, just as we did in the case of our naive Bayesian classifier, and classify the document as ham if the ratio is greater than a threshold, th, and as spam if the ratio is below th. By raising and lowering this threshold we can change the relative importance we place on miss-classified spam and ham messages (see Section 5.1.3).

\subsubsection{Scoring}

The suffix tree representation of a class is richer than the vector representation of more traditional approaches and in developing a scoring method we can experiment with various properties of the tree, each of which can be seen as reflecting certain properties of the class and its members.

We begin by describing how to score a match between a string and a class, then extend this to encompass document scoring. Conceptually dividing the scoring in this way allowed us to introduce and experiment with two levels of normalisation: match-level, reflecting information about strings; and tree-level, reflecting information about the class as a whole. The end of this section elaborates on the underlying motivation for the described scoring method.

\section{Scoring a match}

(a) We define a match as follows: A string $s$ has a match $m=m(s, T)$ in a tree $T$ if there exists in $T$ a path, from the root, $\vec{P}=m$, where $m$ is a prefix of $s$.

(b) The score, $\operatorname{score}(m)$, for a match $m=m_{0} m_{1} m_{2} \ldots m_{n}$, has two parts, firstly, the scoring of each character (and thereby, each substring), $m_{i}$, with respect to its conditional probability, using a significance function of probability, $\phi[\hat{p}]$ (defined below in part(1C)), and secondly, the adjustment (normalisation), $v(m \mid T)$, of the score for the whole match with respect to its probability in the tree:

$$
\operatorname{score}(m)=v(m \mid T) \sum_{i=0}^{n} \phi\left[\hat{p}\left(m_{i}\right)\right]
$$

Using the conditional probability rather than the total probability has the benefit of supporting heuristic H1: as we go deeper down the tree, each node will tend to have fewer children and so the conditional probability will be likely to increase; conversely, there will generally be an increasing number of nodes at each level and so the total probability of a particular node will decrease. Indeed we did experiment with the total probability and found that performance was significantly decreased.

Furthermore, by using the conditional probability we also only consider the independent parts of features when deriving scores. So for example, if $m=$ " $a b c$ ", by the time we are scoring the feature represented by " $a b c$ ", we have already scored the feature " $a b$ ", so we need only score " $c$ " given " $a b$ ".

(c) A function of probability, $\phi[\hat{p}]$, is employed as a significance function because it is not always the most frequently occurring terms or strings which are most indicative of a

\footnotetext{
${ }^{2}$ Diversity is here an intuitive notion which the scoring method attempts to define and represent in a number of different ways.
} 
class. For example, this is the reason that conventional pre-processing removes all stop words, and the most and least frequently occurring terms; however, by removing them completely we give them no significance at all, when we might instead include them, but reduce their significance in the classification decision. Functions on the probability can help to do this, especially in the absence of all pre-processing, but that still leaves the question of how to weight the probabilities, the answer to which will depend on the class.

In the spam domain, some strings will occur very infrequently (consider some of the strings resulting from intra-word characters in the examples of spam in Section 2 above) in either the spam or ham classes, and it is because they are so infrequent that they are indicative of spam. Therefore, under such an argument, rather than remove such terms or strings, we should actually increase their weighting.

Considerations such as these led to experimentation with a number of specifications of the significance function, $\phi[\hat{p}]$ :

$$
\phi[\hat{p}]= \begin{cases}1 & \text { constant } \\ \hat{p} & \text { linear } \\ \hat{p}^{2} & \text { square } \\ \sqrt{\hat{p}} & \text { root } \\ \ln (\hat{p})-\ln (1-\hat{p}) & \text { logit } \\ \frac{1}{1+\exp (-\hat{p})} & \text { sigmoid }\end{cases}
$$

The first three functions after the constant are variations of the linear (linear, sub-linear and super-linear). The last two are variations on the S-curve; we give above the simplest forms of the functions, but in fact, they must be adjusted to fit in the range [0,1].

Although in this paper we are not aiming to develop a probabilistic scoring method, note that the logistic significance function applied to formula (7) may be considered an approximation of such an approach since we generally have a large alphabet and therefore a large number of children at each node, and so for most practical purposes $\ln (1-\hat{p}) \approx 0$.

(d) Turning our attention to match-level normalisation, we experimented with three specifications of $v(m \mid T)$ :

$$
v(m \mid T)= \begin{cases}1 & \text { match unnormalised } \\ \frac{f(m \mid T)}{\sum_{i \in\left(m^{*} \mid T\right)} f(i \mid T)} & \text { match permutation normalised } \\ \frac{f(m \mid T)}{\sum_{i \in\left(m^{\prime} \mid T\right)} f(i \mid T)} & \text { match length normalised }\end{cases}
$$

where $m^{*}$ is the set of all the strings in $T$ formed by the permutations of the letters in $m$; and $m^{\prime}$ is the set of all strings in $T$ of length equal to the length of $m$.

Match permutation normalisation (MPN) is motivated by heuristic H2. The more diverse a class (meaning that it is represented by a relatively large set of substring 
features), the more combinations of characters we would expect to find, and so finding the particular match $m$ is less significant than if the class were very narrow (meaning that it is fully represented by a relatively small set of substring features). Reflecting this, the MPN parameter will tend towards 1 if the class is less diverse and towards 0 if the class is more diverse.

Match length normalisation (MLN) is motivated by examples from standard linear classifiers (see Lewis et al. (1996) for an overview), where length normalisation of feature weights is not uncommon. However, MLN actually runs counter to heuristic H1 because it will tend towards 0 as the match length increases. We would therefore expect MLN to reduce the performance of the classifier; thus MLN may serve as a test of the intuitions governing heuristic $\mathrm{H} 1$.

2. Scoring a document

(a) To score an entire document we consider each suffix of the document in turn and score any match between that suffix and the class tree. Thus the score for a document $s$ is the sum:

$$
\operatorname{SCORE}(s, T)=\sum_{i=0}^{n} \operatorname{score}(s(i), T)
$$

where the $\operatorname{score}(s(i), T)$ searches for a match, $m$, between suffix $s(i)$ and tree $T$, and if one is found, scores it according to formula (7).

We experimented with a number of approaches to tree-level normalisation of the sum in (8) motivated again by heuristic $\mathrm{H} 2$ and based on tree properties such as size, as a direct reflection of the diversity of the class; density (defined as the average number of children over all internal nodes), as an implicit reflection of the diversity; and total and average frequencies of nodes as an indication of the size of the class ${ }^{3}$; but found none of our attempts to be generally helpful to the performance of the classifier. Unfortunately, we do not have space in this paper to further discuss this aspect.

The underlying mechanism of the scoring function can be grasped by considering its simplest configuration: using the constant significance function, with no normalisation. If the scoring method were used in this form to score the similarity between two strings, it would simply count the number of substrings that the two strings have in common. For example, suppose we have a string $t=$ " $a b c d$ ". If we were to apply this scoring function to assessing the similarity that $t$ has with itself, we would obtain a result of 10 , because this is the number of unique substrings that exist in $t$. If we then score the similarity between $t$ and $t^{0}=$ "Xbcd", we obtain a score of 6 , because the two strings share 6 unique substrings; similarly, a string $t^{1}=$ " $a X c d$ " would score 4 .

Another way of viewing this is to think of each substring of $t$ as representing a feature in the class that $t$ represents. The scoring method then weights each of these as 1 if they are present in a query string and 0 otherwise, in a way that is analogous to the simplest form of weighting in algorithms such as Rocchio.

Once seen in this way, we can consider all other flavours of the classifier as experimenting with different approaches to deciding how significant each common substring is, or in other words, deciding how to weight each class feature-much the same as with other non-parametric classifier algorithms.

\footnotetext{
${ }^{3}$ Class size is defined as the total number of substrings in the documents of the class, and tree size as the number of nodes in the tree, that is, the number of unique substrings in the class (see Section 4.3).
} 


\section{Experimental setup}

All experiments were conducted under ten-fold cross validation. We accept the point made by Meyer and Whateley (2004) that such a method does not reflect the way classifiers are used in practice, but the method is widely used and serves as a thorough initial test of new approaches.

We follow convention by considering as true positives (TP), spam messages which are correctly classified as spam; false positives (FP) are then ham messages which are incorrectly classified as spam; false negatives (FN) are spam incorrectly classified as ham; true negatives (TN) are ham messages correctly classified as ham. See Section 5.3 for more on the performance measurements we use.

\subsection{Experimental parameters}

\subsubsection{Spam to ham ratios}

From some initial tests we found that success was to some extent contingent on the proportion of spam to ham in our data set-a point which is identified, but not systematically investigated in other work (Meyer and Whateley, 2004) — and this therefore became part of our investigation. The differing results further prompted us to introduce forms of normalisation, even though we had initially expected the probabilities to take care of differences in the scale and mix of the data. Our experiments used three different ratios of spam to ham: 1:1, 4:6, 1:5. The first and second of these (1:1 and 4:6) were chosen to reflect some of the estimates made in the literature of the actual proportions of spam in current global email traffic. The last of these (1:5) was chosen as the minimum proportion of spam included in experiments detailed in the literature, for example in Androutsopoulos et al. (2000).

\subsubsection{Tree depth}

It is too computationally expensive to build trees as deep as emails are long. Furthermore, the marginal performance gain from increasing the depth of a tree, and therefore the length of the substrings we consider, may be negative. Certainly, our experiments show a diminishing marginal improvement (see Section 6.2.1), which would suggest a maximal performance level, which may not have been reached by any of our trials. We experimented with depths of length of $2,4,6$, and 8 .

\subsubsection{Threshold}

From initial trials, we observed that the choice of threshold value in the classification criterion can have a significant, and even critical, effect on performance, and so introduced it as an important experimental parameter. We used a range of threshold values between 0.7 and 1.3, with increments of 0.1 , with a view to probing the behaviour of the scoring system.

Varying the threshold is equivalent to associating higher costs with either false positives or false negatives because checking that $(\alpha / \beta)>t$ is equivalent to checking that $\alpha>t \beta$. 


\subsection{Data}

\subsubsection{Text corpora}

Three corpora were used to create the training and testing sets:

\section{The Ling-Spam corpus (LS)}

This is available from: http://www.aueb.gr/users/ion/data/lingspam_public. tar.gz. The corpus is that used in Androutsopoulos et al. (2000). The spam messages of the corpus were collected by the authors from emails they received. The ham messages are taken from postings on a public online linguist bulletin board for professionals; the list was moderated, so does not contain any spam. Such a source may at first seem biased, but the authors claim that this is not the case. There are a total of 481 spam messages and 2412 ham messages, with each message consisting of a subject and body.

When comparing our results against those of Androutsopoulos et al. (2000) in Section 6.1 we use the complete data set, but in further experiments, where our aim was to probe the properties of the suffix tree approach and investigate the effect of different proportions of spam to ham messages, we use a random subset of the messages so that the sizes and ratios of the experimental data sets derived from this source are the same as data sets made up of messages from other sources (see Table 1 below).

2. Spam Assassin public corpus (SA)

This is available from: http://spamassassin.org/publiccorpus. The corpus was collected from direct donations and from public forums over two periods in 2002 and

Table 1 Composition of email data sets (EDSs) used in the experiments

\begin{tabular}{|c|c|c|}
\hline EDS Code & $\begin{array}{l}\text { Spam Source } \\
\text { (number from source) }\end{array}$ & $\begin{array}{l}\text { Ham Source } \\
\text { (number from source) }\end{array}$ \\
\hline LS-FULL & LS (481) & LS (2412) \\
\hline LS-11 & LS (400) & LS (400) \\
\hline LS-46 & LS (400) & LS (600) \\
\hline LS-15 & LS (200) & LS (1000) \\
\hline SAe-11 & SAs-G2 (400) & SAe-G2 (400) \\
\hline SAe-46 & SAs-G2 (400) & SAe-G2 (600) \\
\hline SAe-15 & SAs-G2 (200) & SAe-G2 (1000) \\
\hline SAeh-11 & SAs-G2 (400) & SAe-G2 (200) + SAh (200) \\
\hline SAeh-46 & SAs-G2 (400) & SAe-G2 (400) + SAh (200) \\
\hline SAeh-15 & SAs-G2 (200) & SAe-G2 (800) + SAh (200) \\
\hline BKS-LS-11 & BKS (400) & LS (400) \\
\hline BKS-LS-46 & BKS (400) & LS (600) \\
\hline BKS-LS-15 & BKS (200) & LS (1000) \\
\hline BKS-SAe-11 & BKS (400) & SAe-G2 (400) \\
\hline BKS-SAe-46 & BKS (400) & SAe-G2 (600) \\
\hline BKS-SAe-15 & BKS (200) & SAe-G2 (1000) \\
\hline BKS-SAeh-11 & BKS (400) & SAe-G2 (200) + SAh (200) \\
\hline BKS-SAeh-46 & BKS (400) & SAe-G2 (400) + SAh (200) \\
\hline BKS-SAeh-15 & BKS (200) & SAe-G2 (800) + SAh (200) \\
\hline
\end{tabular}


2003, of which we use only the later. The set from 2003 comprise a total of 6053 messages, approximately $31 \%$ of which are spam. The ham messages are split into 'easy ham' (SAe) and 'hard ham' (SAh), the former being again split into two groups (SAe-G1 and SAe-G2); the spam is similarly split into two groups (SAs-G1 and SAs-G2), but there is no distinction between hard and easy. The compilers of the corpus describe hard ham as being closer in many respects to typical spam: use of HTML, unusual HTML markup, coloured text, "spammish-sounding" phrases, etc.

In our experiments we use ham from the hard group and the second easy group (SAe-G2); for spam we use only examples from the second group (SAs-G2). Of the hard ham there are only 251 emails, but for some of our experiments we required more examples, so whenever necessary we padded out the set with randomly selected examples from group G2 of the easy ham (SAe-G2); see Table 1. The SA corpus reproduces all header information in full, but for our purposes, we extracted the subjects and bodies of each; the versions we used are available at: http://dcs.bbk.ac.uk/ ${ }^{\sim}$ rajesh/spamcorpora/spamassassin03.zip

3. The BBKSpam04 corpus (BKS)

This is available at: http://dcs.bbk. ac.uk/ rajesh/spamcorpora/bbkspam04.zip. This corpus consists of the subjects and bodies of 600 spam messages received by the authors during 2004. The Birkbeck School of Computer Science and Information Systems uses an installation of the SpamAssassin filter (apache.org, 2005) with default settings, so all the spam messages in this corpus have initially evaded that filter. The corpus is further filtered so that no two emails share more than half their substrings with others in the corpus. Almost all the messages in this collection contain some kind of obfuscation, and so more accurately reflect the current level of evolution in spam.

One experimental email data set (EDS) consisted of a set of spam and a set of ham. Using messages from these three corpora, we created the EDSs shown in Table 1. The final two numbers in the code for each email data set indicate the mix of spam to ham; three mixes were used: 1:1, 4:6, and 1:5. The letters at the start of the code indicate the source corpus of the set's spam and ham, respectively; hence the grouping. For example, EDS SAe-46 is comprised of 400 spam mails taken from the group SAs-G2 and 600 ham mails from the group SAe-G2, and EDS BKS-SAeh-15 is comprised of 200 spam mails from the BKS data set and 1000 ham mails made up of 800 mails from the SAe-G2 group and 200 mails from the SAh group.

\subsubsection{Pre-processing}

For the suffix tree classifier, no pre-processing is done. It is likely that some pre-processing of the data may improve the performance of an ST classifier, but we do not address this issue in the current paper.

For the the naive Bayesian classifier, we use the following standard three pre-processing procedures:

1. Remove all punctuation.

2. Remove all stop-words.

3. Stem all remaining words.

Words are taken as strings of characters separated from other strings by one or more whitespace characters (spaces, tabs, newlines). Punctuation is removed first in the hope that many of the intra-word characters which spammers use to confuse a Bayesian filter will be removed. 
Our stop-word list consisted of the 57 of the most frequent prepositions, pronouns, articles and conjunctives. Stemming was done using an implementation of Porter's 1980 algorithm, more recently reprinted in Porter (1997). All words less than three characters long are ignored. For more general information on these and other approaches to pre-processing, the reader is directed to Manning and Schutze (1999) and Weiss et al. (2005).

\subsection{Performance measurement}

There are generally two sets of measures used in the literature; here we introduce both in order that our results may be more easily compared with previous work.

Following Sahami et al. (1998), Androutsopoulos et al. (2000), and others, the first set of measurement parameters we use are recall and precision for both spam and ham. For spam (and similarly for ham) these measurements are defined as follows:

$$
\text { Spam Recall }(S R)=\frac{S S}{S S+S H}, \text { Spam Precision }(S P)=\frac{S S}{S S+H S}
$$

where $X Y$ means the number of items of class $X$ assigned to class $Y$; with $S$ standing for spam and $H$ for ham. Spam recall measures the proportion of all spam messages which were identified as spam and spam precision measures the proportion of all messages classified as spam which truly are spam; and similarly for ham.

However, it is now more popular to measure performance in terms of true positive (TP) and false positive (FP) rates:

$$
T P R=\frac{S S}{S S+S H}, \quad F P R=\frac{H S}{H H+H S}
$$

The TPR is then the proportion of spam correctly classified as spam and the FPR is the proportion of ham incorrectly classified as spam. Using these measures, we plot in Section 6 what are generally referred to as receiver operator curves (ROC) (Fawcett, 2004) to observe the behaviour of the classifier at a range of thresholds.

To precisely see performance rates for particular thresholds, we also found it useful to plot, against threshold, false positive rates (FPR) and false negative rates (FNR):

$$
F N R=1-T P R
$$

Effectively, FPR measures errors in the classification of ham and FNR measures errors in the classification of spam.

\section{Results}

We begin in Section 6.1 by comparing the results of the suffix tree (ST) approach to the reported results for a naive Bayesian (NB) classifier on the the Ling Spam corpus. We then extend the investigation of the suffix tree to other data sets to examine its behaviour under different conditions and configurations. To maintain a comparative element on the further data sets we implemented an NB classifier which proved to be competitive with the classifier performance as reported by Androutsopoulos et al. (2000) and others. In this way we look at each experimental parameter in turn and its effect on the performance of the classifier under various configurations. 


\subsection{Assessment}

Table 2 shows the results reported by Androutsopoulos et al. (2000), from the application of their NB classifier on the LS-FULL data set, and the results of the ST classifier, using a linear significance function with no normalisation, on the same data set.

As can be seen, the performance levels for precision are comparable, but the suffix tree simultaneously achieves much better results for recall.

Androutsopoulos et al. (2000) test a number of thresholds (th) and found that their NB filter achieves a $100 \%$ spam precision (SP) at a threshold of 0.001 . We similarly tried a number of thresholds for the ST classifier, as previously explained (see Section 5.1), and found that $100 \%$ SP was achieved at a threshold of 0.98 . Achieving high SP comes at the inevitable cost of a lower spam recall (SR), but we found that our ST can achieve the $100 \%$ in SP with less cost in terms of SR, as can be seen in the table.

As stated in the table (and previously: see Section 5.2.2), we did no pre-processing and no feature selection for the suffix tree. However, both of these may well improve performance, and we intend to investigate this in future work.

As we mentioned earlier (and in Section 3), we use our own NB classifier in our further investigation of the performance of our ST classifier. We therefore begin by presenting in Table 3 the results of this classifier (NB*) on the LS-FULL data set. As the table shows, we found our results were, at least in some cases, better than those reported in Androutsopoulos et al. (2000). This is an interesting result which we do not have space to investigate fully in this paper, but there are a number of differences in our naive Bayes method which may account for this.

Table 2 Results of Androutsopoulos et al. (2000) on the Ling-Spam corpus. In the pre-processing column: 'bare' indicates no pre-processing. The column labelled 'No. of attrib.' indicates the number of word features which the authors retained as indicators of class. Androutsopoulos et al. (2000) quote a 'cost value', which we have converted into equivalent values comparable to our threshold; these converted values are presented, in the ' $t h$ ' column, in a rounded format. Results are shown at the bottom of the table from ST classification using a linear significance function and no normalisation; for the ST classifier, we performed no pre-processing and no feature selection

\begin{tabular}{|c|c|c|c|c|}
\hline Pre-processing & No. of attrib. & $t h$ & $\mathrm{SR}(\%)$ & $\mathrm{SP}(\%)$ \\
\hline \multicolumn{5}{|l|}{ NB } \\
\hline (a) bare & 50 & 1.0 & 81.10 & 96.85 \\
\hline (b) stop-list & 50 & 1.0 & 82.35 & 97.13 \\
\hline (c) lemmatizer & 100 & 1.0 & 82.35 & 99.02 \\
\hline (d) lemmatizer + stop-list & 100 & 1.0 & 82.78 & 99.49 \\
\hline (a) bare & 200 & 0.1 & 76.94 & 99.46 \\
\hline (b) stop-list & 200 & 0.1 & 76.11 & 99.47 \\
\hline (c) lemmatizer & 100 & 0.1 & 77.57 & 99.45 \\
\hline (d) lemmatizer + stop-list & 100 & 0.1 & 78.41 & 99.47 \\
\hline (a) bare & 200 & 0.001 & 73.82 & 99.43 \\
\hline (b) stop-list & 200 & 0.001 & 73.40 & 99.43 \\
\hline (c) lemmatizer & 300 & 0.001 & 63.67 & 100.00 \\
\hline (d) lemmatizer + stop-list & 300 & 0.001 & 63.05 & 100.00 \\
\hline \multicolumn{5}{|l|}{ ST } \\
\hline bare & N/A & 1.00 & 97.50 & 99.79 \\
\hline bare & N/A & 0.98 & 96.04 & 100.00 \\
\hline
\end{tabular}


Table 3 Results of in-house nave Bayes on the LS-FULL data set, with stop-words removed and all remaining words lemmatized. The number of attributes was unlimited, but, for the LS-FULL data set, in practice the spam vocabulary was approximately 12,000 , and the ham vocabulary approximately 56,000 , with 7,000 words appearing in both classes

\begin{tabular}{llllr}
\hline Pre-processing & No. of attrib. & th & SR $(\%)$ & SP(\%) \\
\hline NB* & & & & \\
lemmatizer + stop-list & unlimited & 1.0 & 99.16 & 97.14 \\
lemmatizer + stop-list & unlimited & 0.94 & 89.58 & 100.00 \\
\hline
\end{tabular}

Firstly, Androutsopoulos et al. (2000) use a maximum of 300 attributes, which may not have been enough for this domain or data set, whereas we go to the other extreme of not limiting our number of attributes, which would normally be expected to ultimately reduce performance, but only against an optimal number, which is not necessarily the number used by Androutsopoulos et al. (2000). Indeed, some researchers (Li \& Jain, 1998; Zhang et al., 2004) have found NB does not always benefit from feature limitation, while others have found the optimal number of features to be in the thousands or tens of thousands (Schneider, 2003; McCallum \& Nigam, 1998). Secondly, there may be significant differences in our pre-processing, such as a more effective stop-word list and removal of punctuation; and thirdly, we estimate the probability of word features using Laplace smoothing (see formula (4)), which is more robust than the estimated probability quoted by Androutsopoulos et al. (2000).

There may indeed be further reasons, but it is not our intension in this paper to analyse the NB approach to text classification, but only to use it as a comparative aid in our investigation of the performance of the ST approach under various conditions. Indeed, other researchers have extensively investigated NB and for us to conduct the same depth of investigation would require a dedicated paper.

Furthermore, both our NB* and ST classifiers appear to be competitive with quoted results from other approaches using the same data set. For example, Schneider (2003), experiments on the Ling-Spam data set with different models of NB and different methods of feature selection, and achieves results approximately similar to ours. Schneider (2003) quotes "breakeven" points, defined as the "highest recall for which recall equaled precision", for both spam and ham; Table 4 shows the results achieved by the author's best performing naive Bayes configuration (which we label as ' $\mathrm{NB}^{\prime \prime}$ ) alongside our naive Bayes (NB*) and the suffix tree (ST) using a linear significance function and no normalisation. As can be seen, NB* achieves slightly worse results than the $\mathrm{NB}^{\prime}$, while ST achieves slightly better results; but all are clearly competitive. And as a final example, Surkov (2004) applies developments and extentions of support vector machine algorithms (Vapnik, 1999) to the Ling-Spam data set, albeit in a different experimental context, and achieves a minimum sum of errors of $6.42 \%$; which is slightly worse than the results achieved by our NB* and ST classifiers.

Table 4 Precision-recall breakeven points on the LS-FULL data set

\begin{tabular}{lll}
\hline Classifier & $\operatorname{Spam}(\%)$ & $\operatorname{Ham}(\%)$ \\
\hline $\mathrm{NB}^{\prime}$ & 96.47 & 99.34 \\
$\mathrm{NB}^{*}$ & 94.96 & 98.82 \\
$\mathrm{ST}$ & 98.75 & 99.75 \\
\hline & & 을 Springer
\end{tabular}


Thus, let us proceed on the assumption that both our (NB* and ST) classifiers are at least competitive enough for the task at hand: to investigate how their performance varies under experimental conditions for which results are not available in the literature.

\subsection{Analysis}

In the following tables, we group email data sets (EDSs), as in Table 1, Section 5.2.1, by their source corpora, so that each of the EDSs in one group differ from each other only in the proportion of spam to ham they contain.

\subsubsection{Effect of depth variation}

For illustrative purposes, Table 5 shows the results using the constant significance function, with no normalisation using the LS-11 data set. Depths of 2, 4, 6, and 8 are shown.

The table demonstrates a characteristic which is common to all considered combinations of significance and normalisation functions: performance improves as the depth increases. Therefore, in further examples, we consider only our maximum depth of 8 . Notice also the decreasing marginal improvement as depth increases, which suggests that there may exist a maximal performance level, which was not necessarily achieved by our trials.

\subsubsection{Effect of significance function}

We found that all the significance functions we tested worked very well, and all of them performed better than our naive Bayes. Figure 3 shows the ROC curves produced by each significance function (with no normalisation) for what proved to be one of the most difficult data sets (SAeh-11: see Section 5.2.1).

We found little difference between the performance of each of the functions across all the data sets we experimented with, as can be seen from the summary results in Table 6, which shows the minimum sum of errors (FPR+FNR) achieved at a threshold of 1.0 by each significance function on each data set. The constant function looks marginally the worst performer and the logit and root functions marginally the best, but this difference is partly due to differences in optimal threshold (see Section 6.2.3) for each function: those that perform less well at a threshold of 1.0 may perform better at other thresholds.

Table 7 presents the minimum sum of errors achieved by each function at its individual optimal threshold. In this table there is even less difference between the functions, but still the root is marginally better than the others in that it achieves the lowest average sum of errors over all data sets. And so, for the sake of brevity we favour this function in much of our following analysis.

Table 5 Classification errors by depth using a constant significance function, with no normalisation, and a threhsold of 1 on the LS-11 email data set

\begin{tabular}{lcc}
\hline Depth & FPR(\%) & FNR(\%) \\
\hline 2 & 58.75 & 11.75 \\
4 & 0.25 & 4.00 \\
6 & 0.50 & 2.50 \\
8 & 0.75 & 1.50 \\
\hline
\end{tabular}


All Significance Functions - no normalisation, SAeh-11

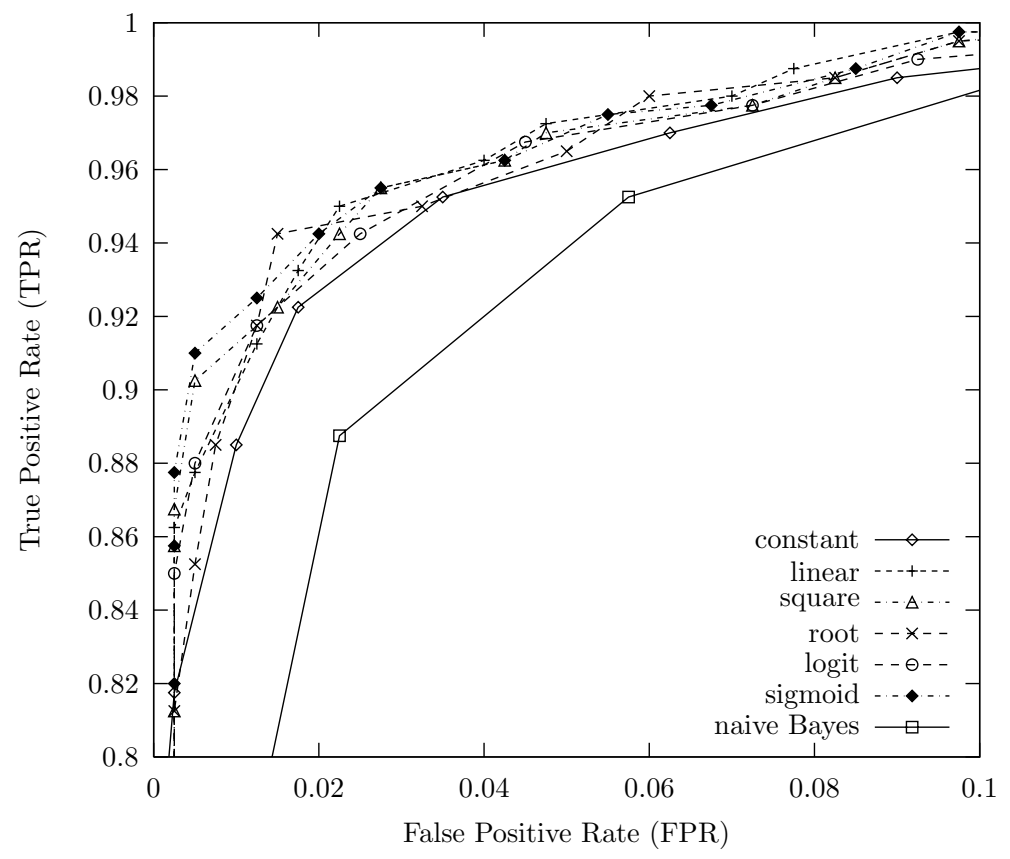

Fig. 3 ROC curves for all significance functions on the SAeh-11 data set

\subsubsection{Effect of threshold variation}

We generally found that there was an optimal threshold (or range of thresholds) which maximised the success of the classifier. As can be seen from the four example graphs shown in Fig. 4, the optimal threshold varies depending on the significance function and the mix of ham and spam in the training and testing sets, but it tends to always be close to 1 .

Obviously, it may not be possible to know the optimal threshold in advance, but we expect, though have not shown, that the optimal threshold can be established during a secondary stage of training where only examples with scores close to the threshold are used-similar to what Meyer \& Whateley (2004) call "non-edge training".

In any case, the main reason for using a threshold is to allow a potential user to decide the level of false positive risk they are willing to take: reducing the risk carries with it an inevitable rise in false negatives. Thus we may consider the lowering of the threshold as attributing a greater cost to miss-classified ham (false positives) than to miss-classified spam; a threshold of 1.0 attributes equal importance to the the two.

Figure 4 Graphs $(\mathrm{a}-\mathrm{c})$ show three different combinations of significance function and data set, the shapes of which are representative of all combinations. The performance of a particular scoring configuration is reflected not only by the minimums achieved at optimal thresholds but also by the steepness (or shallowness) of the curves: the steeper they are, the more rapidly errors rise at sub-optimal levels, making it harder to achieve zero false positives without a considerable rise in false negatives. Graph (d) shows that our NB classifier is the most unstable in this respect. 
Table 6 Sum of errors (FPR+FNR) values at a conventional threshold of 1 for all significance functions under match permutation normalisation (see Section 6.2.4). The best scores for each email data set are highlighted in bold

\begin{tabular}{|c|c|c|c|c|c|c|}
\hline \multirow[b]{2}{*}{ EDS Code } & \multicolumn{6}{|c|}{$\begin{array}{c}\text { Sum of Errors }(\%) \text { at } t h=1 \\
\text { for specifications of } \phi[\hat{p}]\end{array}$} \\
\hline & Constant & Linear & Square & Root & Logit & Sigmoid \\
\hline LS-11 & 1.5 & 2.25 & 2.5 & 1.75 & 1.75 & 1.75 \\
\hline LS-46 & 1.33 & 1.42 & 1.92 & 1.08 & 1.58 & 1.42 \\
\hline LS-15 & 1.33 & 1.33 & 1.55 & 1.33 & 1.55 & 1.89 \\
\hline SAe-11 & 0.25 & 0.5 & 0.5 & 0.5 & 0.25 & 0.75 \\
\hline SAe-46 & 0.5 & 0.75 & 0.5 & 0.5 & 0.25 & 0.75 \\
\hline SAe-15 & 1.00 & 1.50 & 1.8 & 1.5 & 1.1 & 2.00 \\
\hline SAeh-11 & 7.00 & 7.00 & 7.50 & 6.75 & 5.49 & 6.50 \\
\hline SAeh-46 & 4.33 & 4.58 & 4.92 & 5.00 & 4.42 & 4.92 \\
\hline SAeh-15 & 9.3 & 7.5 & 8.00 & 7.7 & 7.6 & 8.6 \\
\hline BKS-LS-11 & 0 & $\mathbf{0}$ & $\mathbf{0}$ & $\mathbf{0}$ & $\mathbf{0}$ & $\mathbf{0}$ \\
\hline BKS-LS-46 & $\mathbf{0}$ & $\mathbf{0}$ & $\mathbf{0}$ & $\mathbf{0}$ & $\mathbf{0}$ & $\mathbf{0}$ \\
\hline BKS-LS-15 & $\mathbf{0}$ & 1.5 & 1.5 & 1.00 & $\mathbf{0}$ & 1.5 \\
\hline BKS-SAe-11 & 4.75 & 1.75 & 1.5 & 1.5 & 1.5 & 1.75 \\
\hline BKS-SAe-46 & 4.5 & 1.75 & 2.00 & 1.75 & 1.5 & 2.75 \\
\hline BKS-SAe-15 & 9.5 & 6.00 & 6.00 & 5.50 & 5.50 & 8.5 \\
\hline BKS-SAeh-11 & 9.25 & 5.75 & 7.25 & 5.00 & 5.75 & 7.25 \\
\hline BKS-SAeh-46 & 10.25 & 5.25 & 7.00 & 4.25 & 5.00 & 7.25 \\
\hline BKS-SAeh-15 & 15.5 & 9.5 & 9.5 & 9.5 & 9.5 & 14.5 \\
\hline
\end{tabular}

\subsubsection{Effect of normalisation}

We found that there was a consistent advantage to using match permutation normalisation, which was able to improve overall performance as well as making the ST classifier more stable under varying thresholds. Figure 5 shows the ROC curves produced by the constant significance function under match permutation normalisation (MPN); match length normalisation (MLN) reduced performance so much that the resulting curve does not even appear in the range of the graph. The stabilising effect of match permutation normalisation is reflected in ROC curves by an increase in the number of points along the curve, but may be better seen in Fig. 6 as a shallowing of the FPR and FNR curves. The negative effect of MLN concurs with our heuristics from Section 4.4. These effects of MPN and MLN were observed, to a greater or lesser degree, for all significance functions and data sets.

\subsubsection{Effect of spam to ham ratios}

We initially found that the mix of spam to ham in the data sets could have some effect on performance, with the degree of difference in performance depending on the data set and the significance function used; however, with further investigation we found that much of the variation was due to differences in the optimal threshold. This can be seen by first examining the differences in performance for different spam:ham ratios shown in Table 6 , in which a 1:5 ratio appears to result in lower performance than the more balanced ratios of $4: 6$ and 1:1; then examining the results presented in Table 7, where differences are far less apparent. These 

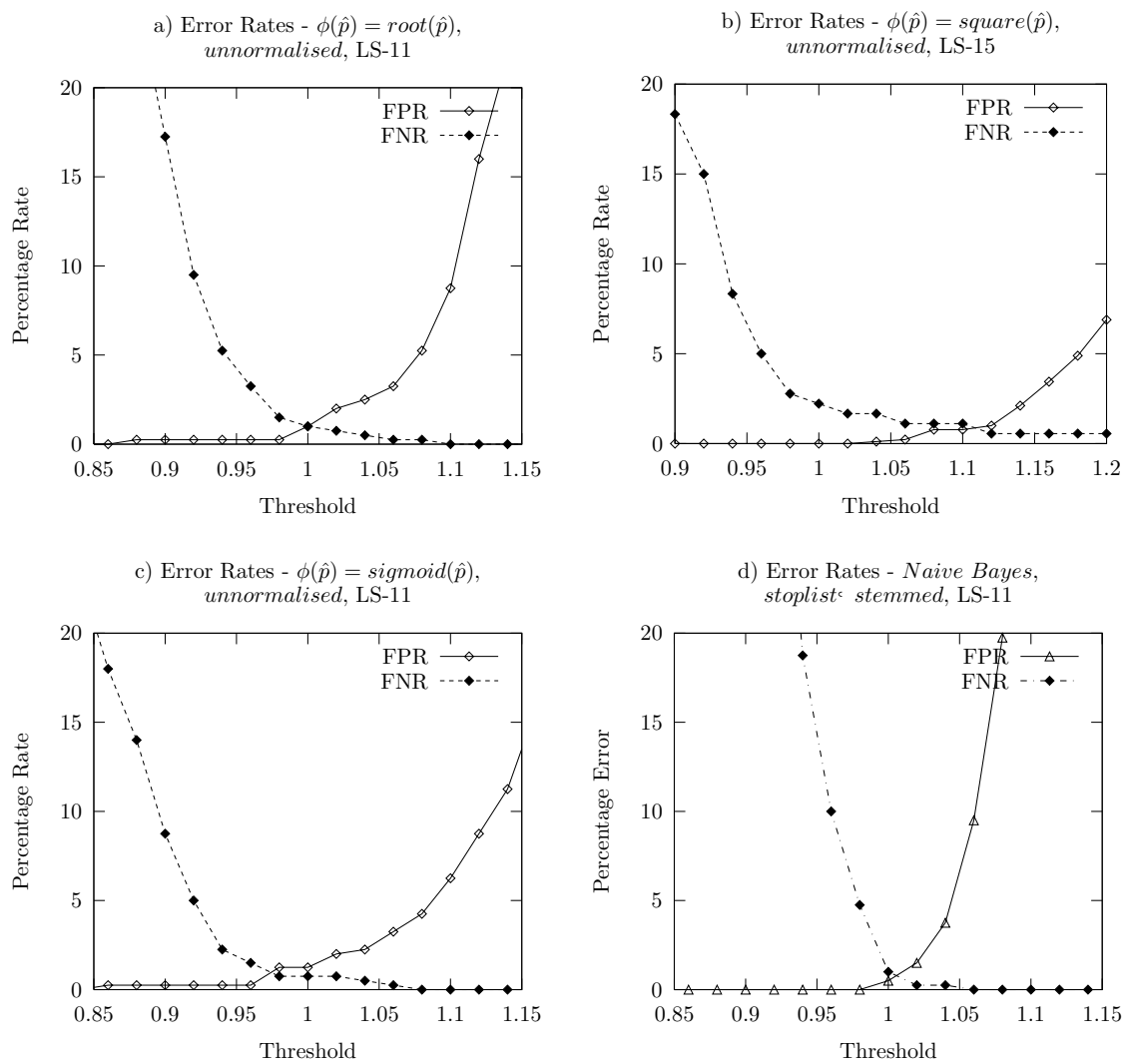

Fig. 4 Effect of threshold variation. Graphs (a-c) show suffix tree false positive (FP) and false negative (FN) rates for three specification of $\phi(\hat{p})$ under no normalisation; graph (d) shows naive Bayes FP and FN rates

observations are reinforced by the graphs shown in Fig. 7. In Graph (a) which shows the ROC curves produced by the constant significance function with no normalisation on the SAeh data sets, we can see that the curves produced by different ratios appear to achieve slightly different maximal performance levels but roughly follow the the same pattern. Graphs (b-d) further show that the maximal levels of performance are achieved at different threshold for each ratio. The difference between Graph (b) and Graph (c) is not great as the difference between the ratios (1:1 and 4:6) is not great; however, the curves shift slightly to the right. The shift is more apparent in Graph (d) because there is a greater change in the ratio (from $1: 1$ to $1: 5)$.

\subsubsection{Overall performance across email data sets}

Table 8 summarises the results for both the ST and NB classifiers at a threshold of 1.0 and Table 9 summarises results at the individual optimal thresholds which minimise the sum of the errors (FPR+FNR).

We found that the performance of the NB is in some cases dramatically improved at its optimal threshold, for example in the case of the BKS-LS data sets. But at both a threshold of 1.0 and at optimal thresholds, the NB classifier behaves very much as expected, supporting our 


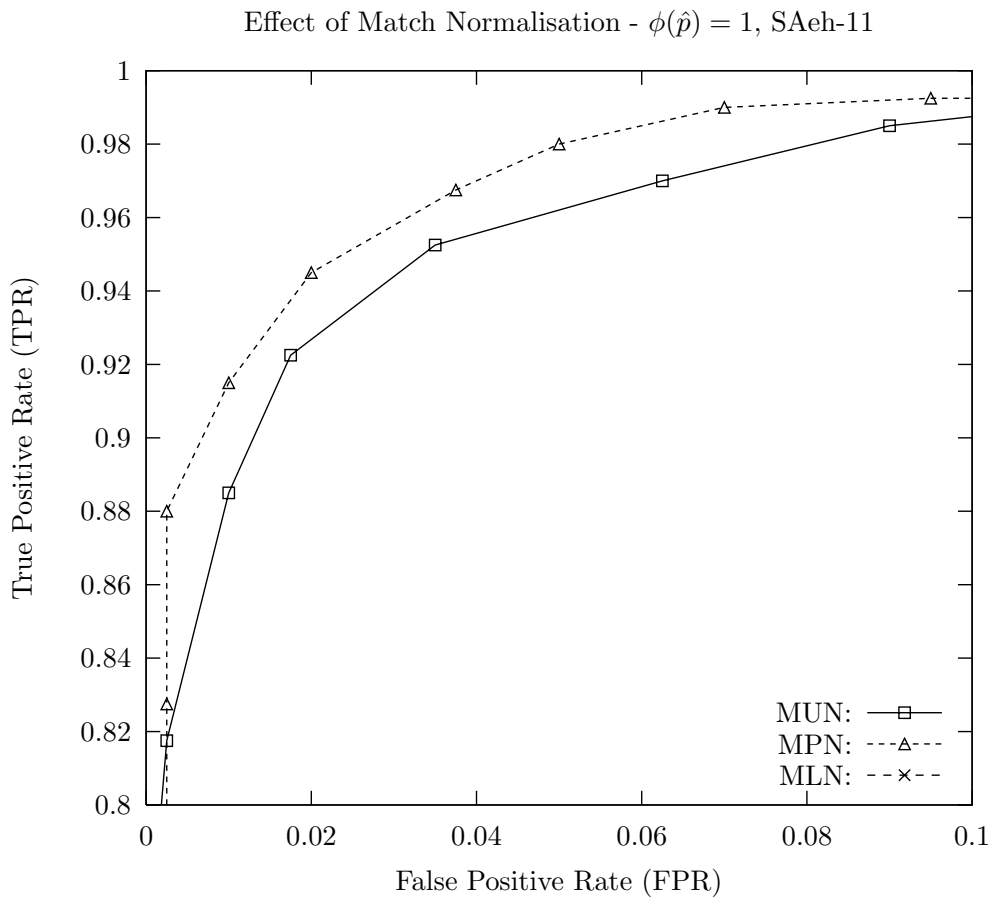

Fig. 5 ROC curves for the constant significance function under no match normalisation (MUN), match permutation normalisation (MPN) and match length normalisation (MLN), on the SAeh-11 data set. MLN has such a detrimental effect on performance that its ROC curve is off the scale of the graph
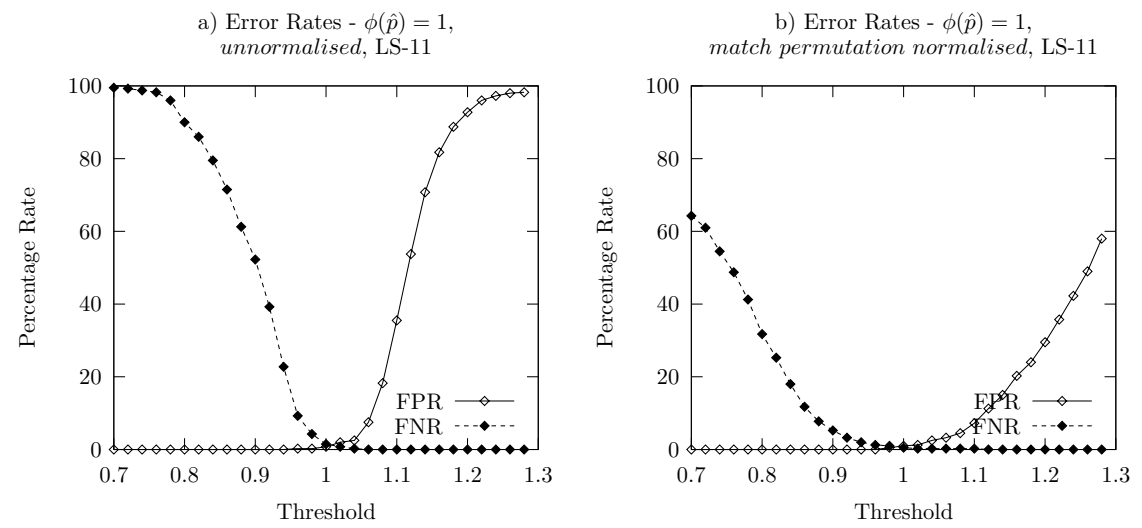

Fig. 6 Effect of match permutation normalisation. False positive (FP) and false negative (FN) rates using a constant significance function on the LS-11 EDS. Graph (a) shows the false positive (FP) and false negative (FN) rates under no normalisation and Graph (b) shows FP and FN rates under match permutation normalisation

initial assumptions as to the difficulty of the data sets. This can be clearly seen in Table 9: on the SAeh data sets which contain ham with 'spammy' features, the NB classifier's false positive rate increases, meaning that a greater proportion of ham has been incorrectly classified as spam; and on the BKS-SAeh data sets which additionally contain spam which is disguised 은 Springer 
Table 7 Sum of error (FPR+FNR) values at individual optimal thresholds for all significance functions under match permutation normalisation (see Section 6.2.4). The best scores for each data set are highlighted in bold

\begin{tabular}{lllllll}
\hline & \multicolumn{7}{c}{$\begin{array}{c}\text { Sum of Errors (\%) at optimal th } \\
\text { for specifications of } \phi[\hat{p}]\end{array}$} \\
\cline { 2 - 7 } EDS Code & Constant & Linear & Square & Root & Logit & Sigmoid \\
\hline LS-11 & 1.25 & $\mathbf{1 . 0 0}$ & $\mathbf{1 . 0 0}$ & $\mathbf{1 . 0 0}$ & $\mathbf{1 . 0 0}$ & $\mathbf{1 . 0 0}$ \\
LS-46 & 1.08 & 1.08 & 1.00 & 1.08 & 1.08 & $\mathbf{0 . 8 3}$ \\
LS-15 & $\mathbf{1 . 3 3}$ & $\mathbf{1 . 3 3}$ & $\mathbf{1 . 3 3}$ & $\mathbf{1 . 3 3}$ & $\mathbf{1 . 3 3}$ & $\mathbf{1 . 3 3}$ \\
SAe-11 & 0.25 & $\mathbf{0}$ & $\mathbf{0}$ & $\mathbf{0}$ & $\mathbf{0}$ & 0.25 \\
SAe-46 & 0.42 & 0.33 & 0.33 & $\mathbf{0 . 2 5}$ & $\mathbf{0 . 2 5}$ & 0.5 \\
SAe-15 & $\mathbf{1 . 0 0}$ & 1.3 & 1.4 & 1.1 & 1.1 & 1.2 \\
SAeh-11 & 7.00 & 6.50 & $\mathbf{6 . 0 0}$ & 6.25 & 6.25 & 6.50 \\
SAeh-46 & $\mathbf{4 . 0 0}$ & 4.58 & 4.92 & 4.42 & 4.33 & 4.92 \\
SAeh-15 & 6.50 & 6.60 & 6.70 & 6.50 & 6.60 & $\mathbf{6 . 3 0}$ \\
BKS-LS-11 & $\mathbf{0}$ & $\mathbf{0}$ & $\mathbf{0}$ & $\mathbf{0}$ & $\mathbf{0}$ & $\mathbf{0}$ \\
BKS-LS-46 & $\mathbf{0}$ & $\mathbf{0}$ & $\mathbf{0}$ & $\mathbf{0}$ & $\mathbf{0}$ & $\mathbf{0}$ \\
BKS-LS-15 & $\mathbf{0}$ & $\mathbf{0}$ & $\mathbf{0}$ & $\mathbf{0}$ & $\mathbf{0}$ & $\mathbf{0}$ \\
BKS-SAe-11 & $\mathbf{0}$ & $\mathbf{0}$ & $\mathbf{0}$ & $\mathbf{0}$ & $\mathbf{0}$ & $\mathbf{0}$ \\
BKS-SAe-46 & $\mathbf{0}$ & $\mathbf{0}$ & $\mathbf{0}$ & $\mathbf{0}$ & $\mathbf{0}$ & $\mathbf{0}$ \\
BKS-SAe-15 & 0.2 & $\mathbf{0}$ & $\mathbf{0}$ & $\mathbf{0}$ & 0.2 & 0.50 \\
BKS-SAeh-11 & 2.75 & $\mathbf{1 . 7 5}$ & 2.00 & $\mathbf{1 . 7 5}$ & 2.00 & 2.00 \\
BKS-SAeh-46 & 1.33 & 1.17 & 1.50 & 1.17 & $\mathbf{1 . 0 0}$ & 1.33 \\
BKS-SAeh-15 & $\mathbf{1 . 1}$ & 1.2 & 2.00 & 1.30 & $\mathbf{1 . 1}$ & 2.1 \\
\hline
\end{tabular}

to appear as ham, the NB classifier's false negative rate increases, meaning that a greater proportion of spam has been misclassified as ham.

The performance of the ST classifier also improves at its optimal thresholds, though not so dramatically, which is to be expected considering our understanding of how it responds to changes in the threshold (see Section 6.2.3). The ST also shows improved performance on data sets involving BKS data. This may be because the character level analysis of the suffix tree approach is able to treat the attempted obfuscations as further positive distinguishing features, which do not exist in the more standard examples of spam which constitute the LS data sets. In all cases except on the SAeh data, the ST is able to keep the sum of errors close to or below $1.0 \%$, and in some cases, it is able to achieve a zero sum of errors. Furthermore, the suffix tree's optimal performance is often achieved at a range of thresholds, supporting our earlier observation of greater stability in its classification success.

\subsubsection{Computational performance}

For illustrative purposes, in this section we provide some indication of the time and space requirements of the suffix tree (ST) classifier using a suffix tree of depth, $d=8$. However, it should be stressed that in our implementation of the ST classifiers we made no attempts to optimise our algorithms as performance was not one of our concerns in this paper. The figures quoted here may therefore be taken as indicators of worst-case performance levels.

Table 10 summarises the time and space requirements of the suffix tree classifier on four of our email data sets. The suffix tree approach clearly and unsurprisingly has high resource 
a) Effect of Spam:Ham ratios - $\phi(\hat{p})=1$, unnormalised

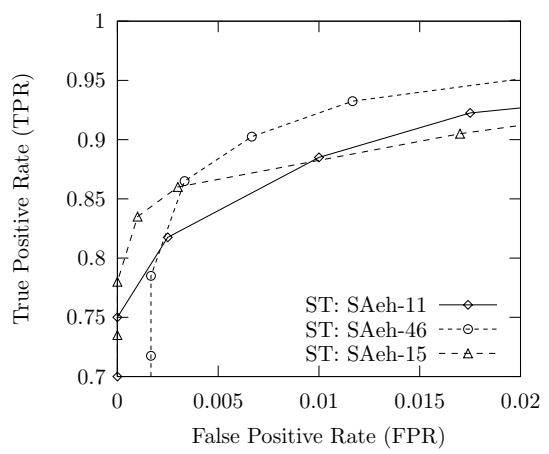

c) Error Rates - $\phi(\hat{p})=1$, unnormalised, SAeh-46

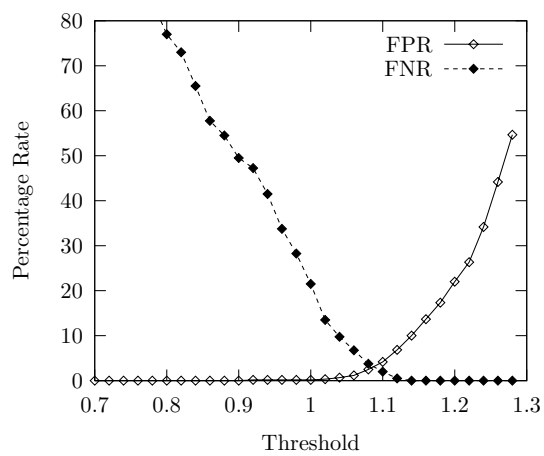

b) Error Rates - $\phi(\hat{p})=1$,

unnormalised, SAeh-11

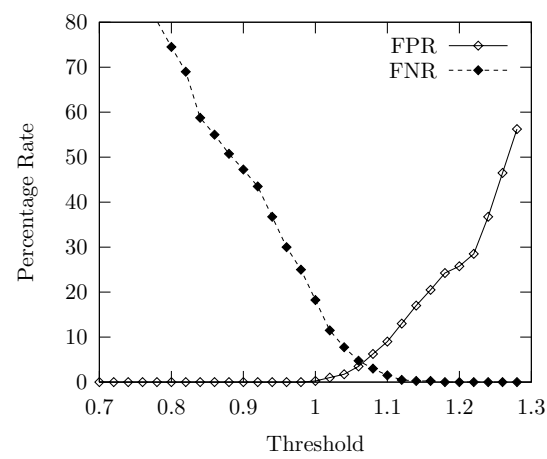

d) Error Rates - $\phi(\hat{p})=1$,

unnormalised, SAeh-15

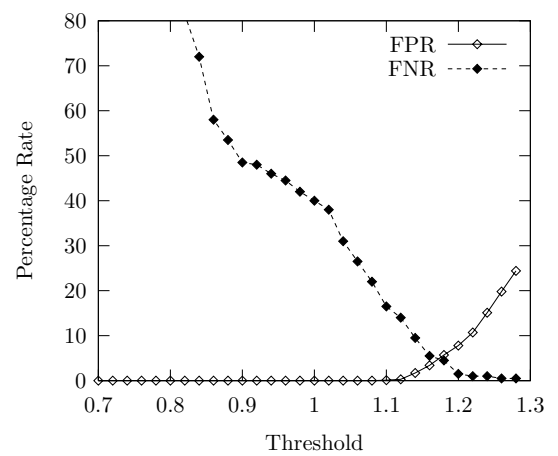

Fig. 7 Effect of varying ratios of spam:ham on the SAeh data using a constant significance function with no normalisation. Graph (a) shows the ROC curves produced for each ratio; while graphs (b-d) show the FP and FN rates separately for ratios of $1: 1,4: 6$ and $1: 5$ respectively

demands, far above the demands of a naive Bayes classifier which on the same machine typically uses no more than $40 \mathrm{MB}$ of memory and takes approximately 10 milliseconds (ms) to make a classification decision.

The difference in performance across the data sets is, however, exactly as we would expect considering our assumptions regarding them. The first point to note is that the mapping from data set size to tree size is non-linear. For example, the LS-FULL EDS is 5 times larger than the LS-11 EDS but results in a tree only 2.95 times larger. This illustrates the logarithmic growth of the tree as more information is added: the tree only grows to reflect the diversity (or complexity) of the training data it encounters and not the actual size of the data. Hence, though the BKS-LS-11 EDS is in fact approximately 25\% smaller than the LS-11 data set, it results in a tree that is over $30 \%$ larger. We would therefore expect to eventually reach a stable maximal size once most of the complexity of the profiled class is encoded.

The current space and time requirements are viable, though demanding, in the context of modern computing power, but a practical implementation would obvious benefit from optimisation of the algorithms. ${ }^{4}$

\footnotetext{
4 The literature on suffix trees deals extensively with improving (reducing) the resource demands of suffix trees (Ukkonen, 1992; Giegerich \& Kurtz, 1997; Kurtz, 1999).
} 
Table 8 Classification errors at threshold of 1 , for Naive Bayes (NB) and a Suffix Tree (ST) using a root significance function and match permutation normalisation

\begin{tabular}{lcclll}
\hline & \multicolumn{2}{c}{ Naive Bayes } & & \multicolumn{2}{c}{ Suffix Tree } \\
\cline { 2 - 3 } \cline { 6 - 6 } EDS Code & FPR $(\%)$ & FNR $(\%)$ & & FPR $(\%)$ & FNR $(\%)$ \\
\hline LS-11 & 1.25 & 0.50 & & 1.00 & 1.75 \\
LS-46 & 0.67 & 1.25 & & 0.83 & 0.25 \\
LS-15 & 1.00 & 1.00 & & 0.22 & 0.13 \\
SAe-11 & 0 & 2.75 & & 0 & 0.50 \\
SAe-46 & 0.17 & 2.00 & & 0 & 0.50 \\
SAe-15 & 0.30 & 3.50 & & 0 & 1.50 \\
SAeh-11 & 10.50 & 1.50 & & 3.50 & 3.25 \\
SAeh-46 & 5.67 & 2.00 & & 2.00 & 3.00 \\
SAeh-15 & 4.10 & 7.00 & & 0.70 & 7.00 \\
BKS-LS-11 & 0 & 12.25 & & 0 & 0 \\
BKS-LS-46 & 0.17 & 13.75 & & 0 & 0 \\
BKS-LS-15 & 0.20 & 30.00 & & 0 & 1.00 \\
BKS-SAe-11 & 0 & 9.00 & & 0 & 1.50 \\
BKS-SAe-46 & 0 & 8.25 & & 0 & 1.75 \\
BKS-SAe-15 & 1.00 & 15.00 & & 0 & 5.5 \\
BKS-SAeh-11 & 16.50 & 0.50 & & 0 & 5.00 \\
BKS-SAeh-46 & 8.17 & 0.50 & & 0 & 4.25 \\
BKS-SAeh-15 & 8.10 & 5.50 & & 0 & 9.50 \\
\hline & & & &
\end{tabular}

Table 9 Classification Errors at optimal thresholds (where the sum of the errors is minimised) for Naive Bayes (NB) and a Suffix Tree (ST) using a root significance function and match permutation normalisation

\begin{tabular}{|c|c|c|c|c|c|c|}
\hline \multirow[b]{2}{*}{ EDS Code } & \multicolumn{3}{|c|}{ Naive Bayes } & \multicolumn{3}{|c|}{ Suffix Tree } \\
\hline & OtpTh & $\operatorname{FPR}(\%)$ & FNR (\%) & OptTh & $\operatorname{FPR}(\%)$ & FNR $(\%)$ \\
\hline LS-11 & 1.0 & 1.25 & 0.50 & 0.96 & 0 & 1.00 \\
\hline LS-46 & 1.02 & 1.00 & 0.67 & 0.96 & 0.33 & 0.75 \\
\hline LS-15 & 1.00 & 1.00 & 1.00 & $0.98-1.00$ & 0.22 & 1.11 \\
\hline SAe-11 & 1.06 & 0.25 & 0 & 1.10 & 0 & 0 \\
\hline SAe-46 & 1.04 & 0.33 & 0.25 & 1.02 & 0 & 0.25 \\
\hline SAe-15 & 1.02 & 2.30 & 1.50 & 1.02 & 0.1 & 1.00 \\
\hline SAeh-11 & 0.98 & 10.50 & 1.50 & 0.98 & 2.75 & 3.50 \\
\hline SAeh-46 & 1.00 & 5.67 & 2.00 & 0.98 & 1.16 & 3.25 \\
\hline SAeh-15 & 1.02 & 7.60 & 1.50 & 1.10 & 3.50 & 3.00 \\
\hline BKS-LS-11 & 1.04 & 0.75 & 2.25 & $0.78-1.22$ & 0 & 0 \\
\hline BKS-LS-46 & 1.06 & 2.50 & 1.25 & $0.78-1.16$ & 0 & 0 \\
\hline BKS-LS-15 & 1.10 & 5.50 & 1.50 & $1.02-1.22$ & 0 & 0 \\
\hline BKS-SAe-11 & $1.04-1.06$ & 0 & 0.25 & $1.04-1.28$ & 0 & 0 \\
\hline BKS-SAe-46 & 1.06 & 0.50 & 0.25 & $1.18-1.28$ & 0 & 0 \\
\hline BKS-SAe-15 & 1.04 & 6.90 & 0 & 1.20 & 0 & 0 \\
\hline BKS-SAeh-11 & 0.98 & 8.00 & 2.00 & 1.06 & 0 & 1.75 \\
\hline BKS-SAeh-46 & 0.98 & 4.00 & 3.75 & $1.14-1.16$ & 0.67 & 0.5 \\
\hline BKS-SAeh-15 & 1.00 & 8.10 & 5.50 & $1.24-1.26$ & 0.80 & 0.50 \\
\hline
\end{tabular}


Table 10 Computational performance of suffix tree classification on four bare (no pre-processing) data sets. Experiments were run on a pentium IV $3 \mathrm{GHz}$ Windows XP laptop with 1 GB of RAM. Averages are taken over all ten folds of cross-validation

\begin{tabular}{lccrc}
\hline EDS Code (size) & Training & AvSpam & AvHam & AvPeakMem \\
\hline LS-FULL (7.40MB) & $63 \mathrm{~s}$ & $843 \mathrm{~ms}$ & $659 \mathrm{~ms}$ & $765 \mathrm{MB}$ \\
LS-11 (1.48MB) & $36 \mathrm{~s}$ & $221 \mathrm{~ms}$ & $206 \mathrm{~ms}$ & $259 \mathrm{MB}$ \\
SAeh-11 (5.16MB) & $155 \mathrm{~s}$ & $504 \mathrm{~ms}$ & $2528 \mathrm{~ms}$ & $544 \mathrm{MB}$ \\
BKS-LS-11 (1.12MB) & $41 \mathrm{~s}$ & $161 \mathrm{~ms}$ & $222 \mathrm{~ms}$ & $345 \mathrm{MB}$ \\
\hline
\end{tabular}

Time could certainly be reduced very simply by implementing, for example, a binary search over the children of each node; the search is currently done linearly over an alphabet of approximately 170 characters (upper- and lower-case characters are distinguished, and all numerals and special characters are considered; the exact size of the alphabet depends on the specific content of the training set). And there are several other similarly simple optimisations which could be implemented.

However, even with a fully optimised algorithm, the usual trade-off between resources and performance will apply. With regard to this, an important observation is that resource demands increase exponentially with depth, whereas performance increases logarithmically. Hence an important factor in any practical implementation will be the choice of the depth of the suffix tree profiles of classes.

\section{Conclusion}

Clearly, the non-parametric suffix tree performs universally well across all the data sets we experimented with, but there is still room for improvement: whereas in some cases, the approach is able to achieve perfect classification accuracy, this is not consistently maintained. Performance may be improved by introducing some pre-processing of the data or postprocessing of the suffix tree profile, and we intend to investigate this in future work. Certainly, the results presented in this paper demonstrate that the ST classifier is a viable tool in the domain of email filtering and further suggests that it may be useful in other domains. However, this paper constitutes an initial exploration of the approach and further development and testing is needed.

In the context of the current work, we conclude that the choice of significance function is the least important factor in the success of the ST approach because all of them performed acceptably well. Different functions will perform better on different data sets, but the root function appeared to perform marginally more consistently well on all the email data sets we experimented with.

Match permutation normalisation was found to be the most effective method of normalisation and was able to improve the performance of all significance functions. In particular it was able to improve the success of the filter at all threshold values. However, other methods of normalisation were not always so effective, with some of them making things drastically worse.

The threshold was found to be a very important factor in the success of the filter. So much so, that the differences in the performances of particular configurations of the filter were often attributable more to differences in their corresponding optimal thresholds than to the configurations themselves. However, as a cautionary note, variations in the optimal threshold may be due to peculiarities of the data sets involved, and this could be investigated further. 
In the case of both the NB and ST filters, it is clear that discovering the optimal threshold - if it were possible - is a good way of improving performance. It may be possible to do this during an additional training phase in which we use some proportion of the training examples to test the filter and adjust the threshold up or down depending on the outcome of each test. Of course, the threshold may be continuously changing, but this could be handled to some extent dynamically during the actual use of the filter by continually adjusting it in the light of any mistakes made. This would certainly be another possible line of investigation.

We also found that the false positive rate (FPR) and false negative rate (FNR) curves created by varying the threshold, were in all cases relatively shallower for our ST classifier than those for our NB classifier, indicating that the former always performs relatively better at non-optimal thresholds, thereby making it easier to minimise one error without a significant cost in terms of the other error.

Finally, any advantages in terms of accuracy in using the suffix tree to filter emails, must be balanced against higher computational demands. In this paper, we have given little attention to minimising this factor, but even though available computational power tends to increase dramatically, cost will nevertheless be important when considering the development of the method into a viable email filtering application, and this is clearly a viable line of further investigation. However, the computational demands of the approach are not intractable, and a suffix tree classifier may be valuable in situations where accuracy is the primary concern.

Acknowledgments The authors are grateful to anonymous referees for thorough comments and suggestions. The authors also wish to thank the Police Information Technology Organisation (PITO) UK for its support.

\section{References}

Aas, K., \& Eikvil, L. (1999). Text categorisation: A survey. Technical report, Norwegian Computing Center. Available online: citeseer.ist.psu.edu/aas99text.html.

Androutsopoulos, I., Koutsias, J., Chandrinos, K., Paliouras, G., \& Spyropoulos, C. (2000). An evaluation of naive bayesian anti-spam filtering. In V. M. G. Potamias and M. van Someren (Eds.), Proceedings of the Workshop on Machine Learning in the New Information Age, 11th European Conference on Machine Learning (ECML 2000), pp. 9-17, Barcelona, Spain.

apache.org. (2005). The apache spamassassin project. Webpage (last accessed November 3, 2005): http://spamassassin.apache.org/index.html.

Bejerano, G., \& Yona, G. (2001). Variations on probabilistic suffix trees: Statistical modeling and prediction of protein families. Bioinformatics, 17(1), 23-43.

de Freitas, S., \& Levene, M. (2004). Spam on the internet: Is it here to stay or can it be eradicated? JISC Technology and Standards Watch Reports (04-01).

Duda, R. O., \& Hart, P. E. (1973). Pattern classification and scene analysis. New York: Wiley-Interscience.

Fawcett, T. (2004). ROC graphs: Notes and practical considerations for researchers. Available online: citeseer.ist.psu.edu/fawcett04roc.html.

Flach, P., \& Lachiche, N. (2004). Naive bayes classification of structured data. Machine Learning, 57(3), 233-269.

Giegerich, R., \& Kurtz, S. (1997). From ukkonen to mccreight and weiner: A unifying view of linear-time suffix tree construction. Algorithmica, 19(3), 331-353.

Graham-Cummings, J. (2004). Spammers compendium. Webpage (last accessed October 20, 2004): http: / / www.jgc.org/tsc/.

Gusfield, D. (1997). Algorithms on strings, trees, and sequences: Computer science and computational biology. Cambridge Unversity Press.

Kurtz, S. (1999). Reducing the space requirement of suffix trees. Software Practice and Experience, 29(13), 1149-1171.

Lewis, D. D. (1998). Naive (Bayes) at forty: The independence assumption in information retrieval. In C. Nédellec and C. Rouveirol (Eds.), Proceedings of ECML-98, 10th European Conference on Machine Learning. (pp. 4-15). Springer Verlag, Heidelberg. 
Lewis, D. D., Schapire, R. E., Callan, J. P., \& Papka, R. (1996). Training algorithms for linear text classifiers. In H.-P. Frei, D. Harman, P. Schäuble, and R. Wilkinson (Eds.), Proceedings of SIGIR-96, 19th ACM International Conference on Research and Development in Information Retrieval. Zürich, CH, (pp. 298306). New York, US: ACM Press.

Li, Y. H., \& Jain, A. K. (1998). Classification of text documents. Comput. J., 41(8), 537-546.

Lloyd, A. (2000). Suffix trees. Webpage (last accessed October 20, 2004): http: / /www . csse. monash. edu.au/ lloyd/tildeAlgDS/Tree/Suffix/.

Lu, B., \& Chen, T. (2003). A suffix tree approach to the interpretation of tandem mass spectra: Applications to peptides of non-specific digestion and post-translational modifications. Bioinformatics, 1990(02), 113ii121.

Manning, C., \& Schutze, H. (1999). Foundations of statistical natural language processing. MIT Press.

McCallum, A., \& Nigam, K. (1998). A comparison of event models for Naive Bayes text classification.

Meyer, T., \& Whateley, B. (2004). SpamBayes: Effective open-source, Bayesian based, email classification system. In Proceedings of the First Conference on Email and Anti-Spam (CEAS). Mountain View, CA. Available online: http: / / www . ceas.cc/papers-2004/136.pdf.

Michelakis, E., Androutsopoulos, I., Paliouras, G., Sakkis, G., \& Stamatopoulos, P. (2004). Filtron: A learningbased anti-spam filter. In Proceedings of the First Conference on Email and Anti-Spam (CEAS). Mountain View, CA. Available online: http: / /www . ceas.cc/papers-2004/142 .pdf.

Porter, M. F. (1997). An algorithm for suffix stripping. In Readings in information retrieval. (pp. 313-316), Morgan Kaufmann Publishers Inc.

Rocchio, J. J. (1971). Relevance feedback in information retrieval. In G. Salton (Ed.), The SMART retrieval system: experiments in automated document processing. (pp. 313-323). Prentice-Hall, Inc.

Sahami, M., Dumais, S., Heckerman, D., \& Horvitz, E. (1998). A bayesian approach to filtering junk e-mail. In Learning for text categorization: papers from the 1998 workshop. Madison, Wisconsin, AAAI Technical Report WS-98-05. Available online: http: / / citeseer.ist.psu.edu/sahami98bayesian. html.

Schneider, K.-M. (2003). A comparison of event models for naive bayes anti-spam e-mail filtering. In Proc. 10th Conference of the European Chapter of the Association for Computational Linguistics (EACL 2003). (pp. 307-314). Budapest, Hungary.

Sebastiani, F. (2002). Machine learning in automated text categorization. ACM Computing Surveys, 34(1), $1-47$.

Surkov, D. (2004). Inductive confidence machine for pattern recognition: Is it the next step towards AI?. Ph.D. thesis, Royal Holloway University of London.

Ukkonen, E. (1992). Constructing suffix-trees on-line in linear time. Algorithms, Software, Architecture: Information Processing, 1(92), 484-492.

unspam.com. (2004). Spam numbers and statistics. Webpage (last accessed October 20, 2004): http: / / www . unspam.com/fight-spam/information/spamstats.html.

Vapnik, V. N. (1999). The nature of statistical learning theory (information science and statistics). Springer.

Weiss, S. M., Indurkhya, N., Zhang, T., \& Damerau, F. J. (2005). Text mining: Predictive methods for analyzing unstructured information. Springer.

Wittel, G. L., \& Wu, S. F. (2004). On attacking statistical spam filters. In Proceedings of the First Conference on Email and Anti-Spam (CEAS). Mountain View, CA. Available online: http: / /www. ceas.cc/ papers-2004/170.pdf.

Zhang, L., Zhu, J., \& Yao, T. (2004). An evaluation of statistical spam filtering techniques. ACM Transactions on Asian Language Information Processing (TALIP), 3(4), 243-269. 